\section{FreiLacke ist nationaler Sieger bei Wassermanagement}

Einest ne Jury aus Vertretern des Bundesumweltministeriums, des Umweltbundesamts, der Deutschen Akkreditierungsgesellschaft für Umweltgutachter, des Umweltgutachterausschusses und des Deutschen Industrie- und Handelskammertages hat FreiLacke für den Emas-Award 2012 nominiert. Der Lackhersteller tritt damit am 29. November in Brüssel als Vertreter von Deutschland in der Kategorie "Große Organisationen" gegen die Nominierten der übrigen EU-Mitglieder um den EmasAward 2012 an. Der Award ist die renommierteste Auszeichnung für ein betriebliches Umweltmanagement. 1996 nahm FreiLacke als erste Lackfabrik in Deutschland an Emas teil und konnte in der Zwischenzeit beachtliche Erfolge vorweisen: So reduzierte das Unternehmen seinen Frischwasser-Verbrauch zwischen 1995 und 2011 von über 15700 Kubikmeter auf nur noch 3300 Kubikmeter bei nahezu verdoppelter Produktionsmenge. Mit der Regenwassernutzung reduzierte der Lackhersteller nicht nur den Wasser-Verbrauch, sondern verringerte auch die Energie- und Lösemittelverbräuche, vor allem in den Prozessen Kühlkreislauf und Behälterreinigung.
Im Prozess Kühlkreislauf konnten über $2.000 .000 \mathrm{kWh}$ eingespart werden. Die Lösemittelmengen für die Behälterreinigung reduzierten sich um fast 80 Tonnen Lösemitteln pro Jahr. Sogar das bisherige Lösemittel Ethylacetat als Spülmedium konnte durch Regenwasser ersetzt werden. Selbst der Löschwasservorrat für die Sprinkleranlage der Hochregallager wird mit Regenwasser gespeist. Die Modernisierung der Heiztechnik mit Wasser-Wärmepumpen und Wärmetauschern sorgt für eine Kühlung im Sommer und eine angenehme Arbeitstemperatur im Winter. Bis zu einer Außentemperatur von $0^{\circ} \mathrm{C}$ ist eine Zusatzheizung mit Öl nicht notwendig. Damit senkte sich der Energieverbrauch der Heizung um 66 Prozent und die dadurch bedingten Emissionen um 64 Prozent. In Sachen Umweltschutz betreibt der Lackhersteller aktive Kommunikationsarbeit. Neben weiteren Optimierungen im Wassermanagement wird zukünftig ein Schwerpunkt im Unternehmen die Verbesserungen der Energieeffizienz einnehmen. Hierfür sind die Vorbereitungen im Energiemanagementsystem nach DIN EN ISO 50001 bereits umgesetzt.

\section{Investorengruppe übernimmt Fischer- Draht GmbH}

Rückwirkend zum 1. Oktober 2012 Thaben Peter Borst und Martin Stiefel die Fischer-Draht GmbH - ehemals Heinrich Fischer GmbH \& Co. KG - übernommen. Die neue Investorengruppe steht zum Standort Winterlingen und wird einen Großteil der heutigen Arbeitnehmer übernehmen. Durch die Übernahme des Markennamens „FischerDraht" soll zudem verdeutlicht werden, dass es sich hierbei nicht um einen Neuanfang sondern um die erfolgreiche Weiterführung der Marke und damit auch um die Fortführung des traditionellen Produktprogrammes geht. Laut Peter Borst wird der Produktbereich „Industrie + Handel“ übernommen. Hierzu zählen Premium-Wasch- und Warenkörbe, Werkstückträgersysteme, Container- und Regalkorbsysteme, Lager- und Transportkörbe sowie Kundenführungssysteme. Zusätzlich wird ein neuer Geschäftsbereich für Logistiksysteme, wie zum Beispiel Kistenhubgeräte, aufgebaut mit dem Ziel, zukünftig als Komplettlösungsanbieter im Bereich „Logistik" und "Logistikprozess" auftreten zu können. Die Kunden sollen dabei mit Unterstützung der Fischer-Draht GmbH ihre Kosten für den Prozess reduzieren sowie die Effektivität steigern können.

\title{
Studie belegt hohe Ökoeffizienz bei Integrierten Prozessen
}

De er Lackierprozess ist einer der energieintensivsten Prozessschritte bei der industriellen Automobilfertigung. Freigesetzte Lösemittel, Energieverbrauch oder $\mathrm{CO}_{2}$ Bilanz spielen eine bedeutende Rolle. In einer neuen umfangreichen Analyse von BASF und Dürr wurde die Ökoeffizienz von Anlagen- und Lacktechnik ganzheitlich untersucht. Die von BASF entwickelte Ökoeffizienz-Analyse setzt die Wirtschaftlichkeit von Produkten und Prozessen ins Verhältnis zu ihren Auswirkungen auf die Umwelt. Sie soll bei der Entscheidung helfen, welche Produkte und Prozesse sowohl wirtschaftlich als auch ökologisch am besten geeignet sind. Dazu wird der gesamte Lebensweg eines Produkts oder Verfahrens ganzheitlich betrachtet, also von der Rohstoffgewinnung über die Herstellung und Verwendung des Produkts bis zur Entsorgung. Die wichtigsten Ergebnisse der aktuellen Studie beziehen sich auf Lackierprozesse mit Füller, verkürzte Lackierprozesse sowie auf den Einsatz moderner Anlagen- und Applikationstechnik. Bei den Prozessen mit Füllern haben BASF und Dürr die wichtigsten industrieüblichen Varianten von lösemittelhaltigen und wasserbasierten Lacksystemen untersucht. Die Studie ergab, dass wasserbasierte Prozesse bei der Bewertung ihrer Ökoeffizienz insgesamt gut abschneiden. Der Vergleich der Ökoeffizienz von herkömmlichen Füllerprozessen mit integrierten Prozessen fiel in Summe eindeutig zugunsten der integrierten Prozesse aus. Deutliche Einsparpotenziale ergeben sich beim Energieverbrauch der Lackierkabinen, wenn diese in Umluftfahrweise betrieben werden. Die Studie bezieht sich nicht auf konkrete Werte. Vielmehr wird die Vergleichbarkeit der Werte dadurch ermöglicht, dass realistische Annahmen zu Verbräuchen, Emissionen und Kosten gemacht wurden. 